TITLE:

\title{
Energy distribution of the compact microwave ion source for extremely low voltage ion extraction
}

\section{$\operatorname{AUTHOR}(\mathrm{S})$ :}

Gotoh, Y; Nakajima, K; Hagiwara, T; Tsuji, H; Ishikawa, J

\section{CITATION:}

Gotoh, Y ... [et al]. Energy distribution of the compact microwave ion source for extremely low voltage ion extraction. REVIEW OF SCIENTIFIC INSTRUMENTS 2002, 73(2): 758-760

\section{ISSUE DATE:}

2002-02

URL:

http://hdl.handle.net/2433/39805

\section{RIGHT:}

Copyright 2002 American Institute of Physics. This article may be downloaded for personal use only. Any other use requires prior permission of the author and the American Institute of Physics. 


\title{
Energy distribution of the compact microwave ion source for extremely low voltage ion extraction
}

\author{
Y. Gotoh, ${ }^{a}$ K. Nakajima, T. Hagiwara, H. Tsuji, and J. Ishikawa \\ Department of Electronic Science and Engineering, Kyoto University, Yoshida-honmachi, Sakyo-ku, \\ Kyoto 606-8501, Japan
}

(Presented on 4 September 2001)

\begin{abstract}
The energy distribution of the ion beam extracted from the compact microwave ion source for extremely low voltage ion extraction was measured with $\mathrm{Ar}$ and $\mathrm{CO}$ as a discharge gas. The energy distribution was measured by the retarding field method, and changes with respect to the change in gas pressure were observed. The obtained data were arranged by the peak energy and the energy spread. For both gases, the peak energy and the energy spread decreased with an increase in the gas pressure. The energy spread of approximately $5 \mathrm{eV}$ with the peak energy of $15 \mathrm{eV}$ were obtained for $\mathrm{Ar}$ gas at the pressure of $10^{-2} \mathrm{~Pa}$. For $\mathrm{CO}$ gas, the peak energy was higher than $\mathrm{Ar}$ and approximately $20 \mathrm{eV}$. The energy spread was $6 \mathrm{eV}$ at the pressure of $10^{-2} \mathrm{~Pa}$. These values agreed with the peak energy and energy spread that were estimated previously from the mass spectra analysis. Since the ion source was designed to be used in the researches of low energy ion-solid interaction, these characteristics satisfy the requirements for this purpose. (C) 2002 American Institute of Physics. [DOI: 10.1063/1.1429781]
\end{abstract}

\section{INTRODUCTION}

We have developed a compact microwave ion source, which can provide extremely low energy ions for the purpose of the researches of low energy ion-solid interaction. ${ }^{1,2}$ The energy range of interest is $10-200 \mathrm{eV}$, which causes a significant effect for thin film formation. In order to produce such a low energy ion beam, the ion beam is generally once accelerated to some tens of $\mathrm{keV}$ and then decelerated to have the low energy. In such a case, neutralized fast atoms are incident on the sample, and analysis of the effect of ion irradiation to the solid surface becomes difficult. We are developing the ion source which does not require preacceleration, that is, the source which yield sufficient ion current at the extraction voltage which corresponds to the final ion energy. We modified the compact microwave ion source with a permanent magnet ${ }^{3}$ to meet the extraction at the extremely low voltage. As we showed in our previous article, ${ }^{2}$ the argon (Ar) ion beam extracted from the modified ion source exhibited well separated $\mathrm{Ar}^{2+}$ and $\mathrm{Ar}^{+}$peaks in the mass spectrum, even at the extraction voltage of $4 \mathrm{~V}$. This would be attributed to the fact that the energy spread of the ion beam is narrow enough to be separated by a small sector magnet. From the separation of the peaks of $\mathrm{Ar}^{2+}$ and $\mathrm{Ar}^{+}$, we estimated that the energy spread would be narrower than $12 \mathrm{eV}$. We also analyzed the peak position of the mass spectrum and estimated that the plasma potential was between 14 and $66 \mathrm{eV}$ in the gas pressure range of $(0.75-8.2)$ $\times 10^{-3} \mathrm{~Pa}$. We are planning to apply this ion source to the research of ion-solid interaction. From this point of view, this narrow energy spread is preferable to determine the ion en-

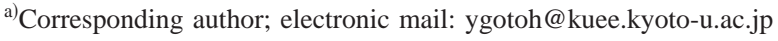

ergy as well as to transport the ion beam efficiently. Determining the absolute ion energy including plasma potential is also necessary.

From the above point of view, we measured the energy distribution of the ion beam extracted from the ion source as a function of some operational parameters.

\section{EXPERIMENTAL SETUP}

Figure 1 shows the schematic diagram of the compact microwave ion source for extremely low voltage ion extraction. The ion source used was exactly the same as that described in the previous article. ${ }^{2}$ The microwave is supplied through the coaxial $\mathrm{N}$-type connector and is radiated by an antenna. The most prominent feature of the present source is having a cone-cave plasma chamber, in order to make the plasma thinner at the extraction region, to meet the low voltage ion extraction. The ion source was mounted on a test bench which was pumped by a turbomolecular pump with the nominal pumping speed of $801 \mathrm{~s}^{-1}$. The background pressure was about $10^{-4} \mathrm{~Pa}$. As the discharge gas, we selected $\mathrm{Ar}$ and $\mathrm{CO}$. The ion source could be operated at the pressure of $1 \times 10^{-3} \mathrm{~Pa}$ to $1 \times 10^{-2} \mathrm{~Pa}$. The ion extraction was performed with a single aperture system. The diameter of the aperture was $0.5 \mathrm{~mm}$.

The energy distribution of the extracted beam was measured by a retarding field method, designated for the present measurement. The extracted ion beam has a relatively large divergence due to space charge effect, and a common retarding field analyzer may not work well. In the present analyzer, deceleration of the divergent beam is made so as not to give a lateral velocity component to the ions, resulting in better energy resolution. The detailed description of the present energy analyzer will be shown elsewhere, ${ }^{4}$ and here we explain the analyzer briefly. The analyzer is composed of three elec- 


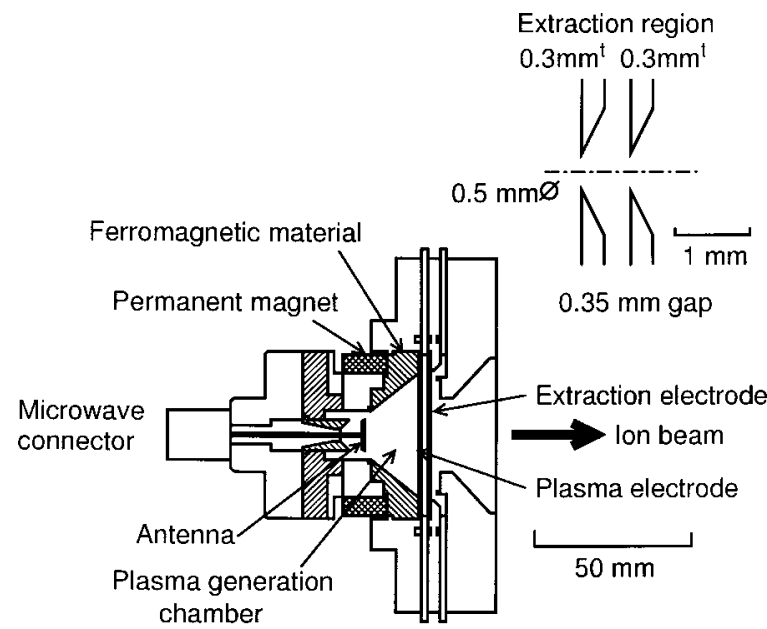

FIG. 1. Schematic diagram of the compact microwave ion source for extremely low voltage ion extraction.

trodes, a beam limiter, a suppressor, and a retarding collector. The collector has a hemispherical mesh electrode. Since the acceleration voltage is low enough, an ammeter can be floated. Applying the voltage to the curved collector, the retarding field becomes inward. Even if the ion has a large lateral velocity component at the exit of the suppressor, the electric force may act on the ion along its motion.

By applying negative potential to the suppressor electrode, we can eliminate low energy secondary electrons flowing to the collector. Controlling this voltage, it is possible to adjust the object position.

The measurements of the energy distribution were promoted with argon (Ar) and carbon monoxide (CO) gases. The measurement was done just after the ion extraction, and thus the ion beam was not mass separated. The energy distribution curves were measured at different operating gas pressures between $10^{-3} \mathrm{~Pa}$ and $10^{-2} \mathrm{~Pa}$. The pressure was measured by the vacuum gauge equipped at the position above the pumping system. The correction factors for $\mathrm{Ar}$ (1.21) and $\mathrm{CO}(1.04)^{5}$ were taken into consideration. The pressure in the discharge chamber was estimated to be 7-70 $\mathrm{Pa}$ from the conductance and the pumping speed of the system, assuming the molecular flow. The pressure may be slightly lower than the above value because of the effective pumping speed is lower than $801 \mathrm{~s}^{-1}$, and also the above

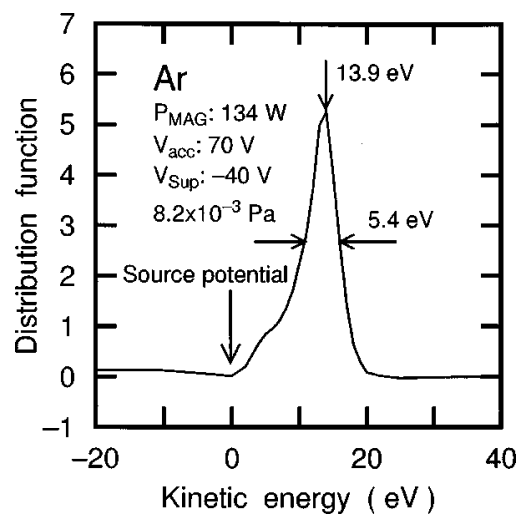

FIG. 2. Typical example of the energy distribution of Ar ion beam.

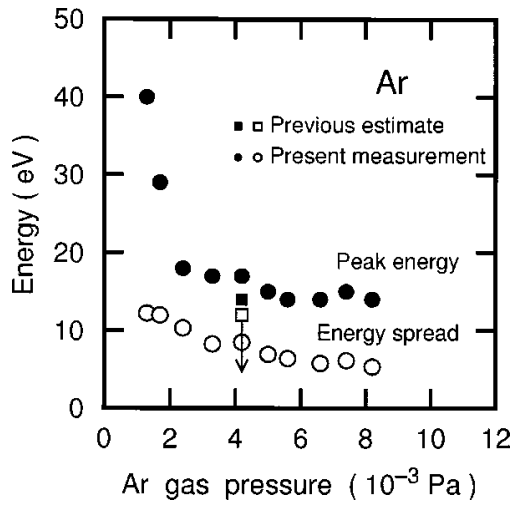

FIG. 3. Peak energy and energy spread of Ar ion beam as a function of the gas pressure.

pressure range is intermediate flow region, resulting in increase of the conductance. The ion extraction voltage was $60-70 \mathrm{~V}$ and the suppressor voltage was -20 to $-40 \mathrm{~V}$.

\section{RESULTS}

Figure 2 shows the typical example of the energy distribution curve for the Ar ion beam operated at the pressure of $8.2 \times 10^{-3} \mathrm{~Pa}$. The origin of the abscissa is taken for the ion source potential. The distribution has a maximum at the energy of $13.9 \mathrm{eV}$ above the source potential. The full width at half maximum of the energy distribution curve is about 5.4 $\mathrm{eV}$. Here we define the peak energy as that shows the maximum of the energy distribution curve, with respect to the source potential. Also we define the energy spread by the full width at half maximum of the curve.

These characteristic values varied with the operating pressure. Figure 3 shows the peak energy and the energy spread as a function of operating Ar gas pressure. The solid circle and the open circle show the peak energy and the energy spread, respectively. The magnetron input was $134 \mathrm{~W}$. As the gas pressure increases, the energy spread decreased and the peak energy also decreased. The change was relatively small at the higher gas pressure, but at the pressure lower than $10^{-3} \mathrm{~Pa}$, changes in the peak energy becomes large. In the figure, the values of the peak energy and the energy spread estimated in the previous report ${ }^{2}$ are also shown by the solid square and the open square, respectively.

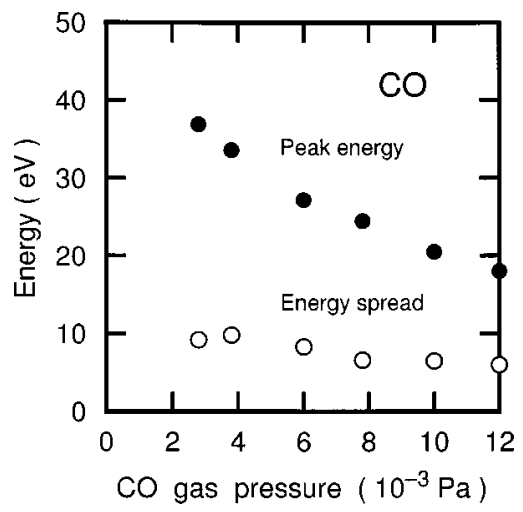

FIG. 4. Peak energy and energy spread of $\mathrm{CO}$ ion beam as a function of the gas pressure. 
The estimation of the peak energy was done by plotting the relation between ion acceleration voltage and square of the exiting current of the mass separator. The estimation of the energy spread was done by the necessary condition for separation of $\mathrm{Ar}^{2+}$ and $\mathrm{Ar}^{+}$under the given condition. The latter gives the highest limit and the actual energy spread may be narrower than this estimated value.

These estimated values coincided with those obtained in the present study. Also the extrapolated peak energy curve obtained in the present study coincided the previously estimated value of $66 \mathrm{eV}$ at the Ar pressure of $0.75 \times 10^{-3} \mathrm{~Pa}$.

Quite similar results were observed for $\mathrm{CO}$ gas. Figure 4 shows the peak energy and the energy spread as a function of the operating $\mathrm{CO}$ gas pressure. The magnetron input power was $104 \mathrm{~W}$. The peak energy gradually decreased with an increase in the gas pressure, and almost $20 \mathrm{eV}$ at the pressure of $1.2 \times 10^{-2} \mathrm{~Pa}$. The change in the peak energy was larger than that for Ar. The energy spread is almost the same with that of Ar.

\section{DISCUSSION}

The decrease of the peak energy, which is equivalent to the plasma potential, with an increase in the operating gas pressure is explained in the following manner. As the gas pressure increases, the collision frequency between the electrons and the neutral gas atoms increases. The electrons could not gain the energy from the microwave, resulting in the lowering of the electron temperature. Since the plasma potential is dominated by the electron temperature, ${ }^{6}$ the plasma potential decreases with an increase in the gas pressure.

For CO gas, the energy of the electrons is dissipated for dissociation of the $\mathrm{CO}$ molecule, and thus generally electron temperature would be lower than noble gas case. However, the ionization power of low energy electrons for $\mathrm{CO}$ gas is lower than that for Ar gas. ${ }^{7}$ This may result in higher electron temperature for $\mathrm{CO}$ gas.

The present results suggested that the operation at the higher pressure is preferred. However, operation at the pressure $10^{-2} \mathrm{~Pa}$ is not suitable in use for ultrahigh vacuum systems. It is necessary to use a pump with a larger pumping speed.

\section{SUMMARY}

The energy distribution of the compact microwave ion source for extremely low voltage ion extraction was measured. The energy spread was as low as $5 \mathrm{eV}$. The energy distribution of the mass-separated ion beam should be measured in the near future. The narrow energy spread enabled us to transport the low energy ion beam effectively. We have recently constructed the extremely low energy ion irradiation system for in situ observation of scanning tunneling microscope. ${ }^{8}$ The operation at the relatively high pressure gives a stable operation of peak energy and energy spread. Similar experiments for the mass-separated ion beam are also undergoing.

${ }^{1}$ Y. Gotoh, Y. Fujimori, H. Kubo, H. Tsuji, and J. Ishikawa, Rev. Sci. Instrum. 69, 887 (1998)

${ }^{2}$ Y. Gotoh, H. Kubo, H. Tsuji, and J. Ishikawa, Rev. Sci. Instrum. 71, 1160 (2000).

${ }^{3}$ J. Ishikawa, Y. Takeiri, and T. Takagi, Rev. Sci. Instrum. 55, 449 (1984).

${ }^{4}$ Y. Gotoh, K. Nakajima, H. Tsuji, and J. Ishikawa (in preparation).

${ }^{5}$ Vacuum Society of Japan, Kansai Division, Ed., Introduction to the Vacuum Technology, 2nd ed. (Nikkan Kogyo Shinbun, Tokyo, 1998) [in Japanese].

${ }^{6}$ B. N. Chapman, Glow Discharge Processes (Wiley, New York, 1980).

${ }^{7}$ A. von Engel, Ionized Gases (Oxford University Press, Oxford, 1965).

${ }^{8}$ Y. Gotoh, T. Hagiwara, Y. Tanaka, H. Kubo, H. Tsuji, and J. Ishikawa, Mater. Sci. Eng., A (submitted). 